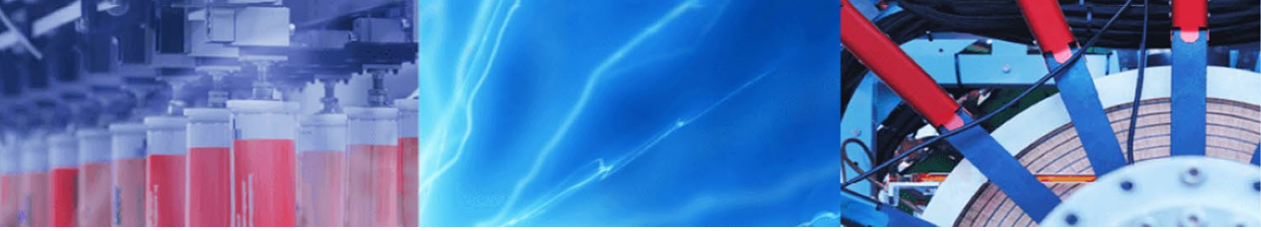

Research Article

\title{
Selective adsorption of carbon dioxide from mixed vapors by blockage of methane in graphene nanoribbons
}

\author{
Hind Aljaddani ${ }^{1} \cdot$ Silvina M. Gatica ${ }^{1}$ (I)
}

Received: 28 November 2019 / Accepted: 6 March 2020 / Published online: 16 March 2020

(c) Springer Nature Switzerland AG 2020

\begin{abstract}
We study numerically the adsorption of a mixture of $\mathrm{CO}_{2}$ and $\mathrm{CH}_{4}$ on a graphite substrate covered by graphene nanoribbons (NRs). The NRs are flat and parallel to the graphite surface, at a variable distance ranging from 6 to $14 \AA$ A. We show that the NRs-graphite substrate acts as an effective filter for $\mathrm{CO}_{2}$. Our study is based on molecular dynamics simulations. Methane is considered a spherical molecule, and carbon dioxide is represented as a linear rigid body. Graphite is modeled as a continuous material, while the NRs are approached atomistically. We observe that when the NRs are placed 6 $\AA$ above the graphite surface, methane is blocked out, while $\mathrm{CO}_{2}$ molecules can diffuse and be collected in between the $\mathrm{NRs}$ and the graphite surface. Consequently, the selectivity of $\mathrm{CO}_{2}$ is extremely high. We also observe that the initial rate of adsorption of $\mathrm{CO}_{2}$ is much higher than $\mathrm{CH}_{4}$. Overall we show that the filter can be optimized by controlling the gap between NRs and the NRs-graphite separation.
\end{abstract}

Keywords Gas separation · Graphene nanoribbons · Carbon dioxide · Methane · Adsorption

\section{Introduction}

Separating $\mathrm{CO}_{2}$ from $\mathrm{CH}_{4}$ is critical in industrial applications, transportation, and usage of methane [1]. Researchers have intensively investigated the adsorption of $\mathrm{CO}_{2}$ $/ \mathrm{CH}_{4}$ mixtures on several materials like MOFs, mesoporous carbon, activated carbon, silicalite, $\mathrm{C}_{186}$ schwarzite, and nanoporous carbon experimentally or theoretically [1-9].

Gas separation by adsorption can be accomplished by three physical mechanisms: equilibria, kinetics, and steric effects. Equilibrium mechanisms rely on the strength of attraction between gas molecules and the substrate, while kinetic mechanisms involve the differences in the adsorption and transport rates of gas on and through the substrate [3]. Steric mechanisms, on the other hand, depend on the incompatibility between the size or shape of the adsorbate gas molecules and the pores of the substrate. For instance, since $\mathrm{CO}_{2}$ is typically found in a mixture with gases of similar size but different shapes (as $\mathrm{CH}_{4}$ and $\mathrm{N}_{2}$ ), steric separation may be feasible. Also, the force of some substrates is stronger to $\mathrm{CO}_{2}$ than methane. For example, in this study, we found that the energy of interaction of $\mathrm{CO}_{2}$ with graphite is $35 \%$ stronger than $\mathrm{CH}_{4}$ with graphite. As a result, the equilibrium mechanism may also present an adequate strategy.

The ability of a substrate to separate gases by adsorption is measured by the selectivity. In a binary mixture of components $i$ and $j$, the selectivity is defined as,

$$
\Sigma(i / j)=\frac{x_{i} / x_{j}}{y_{i} / y_{j}}
$$

where $x_{i}$ and $y_{i}$ are the molar concentration of species $i$ in the adsorbed phase and the vapor phase respectively. A high value of $\Sigma(i / j)$ indicates that the fraction of species $i$ in the adsorbed phase is large compared to the fraction in the vapor phase, meaning that $i$ is favorably adsorbed.

Silvina M. Gatica, sgatica@howard.edu; Hind Aljaddani, hind.aljaddani@howard.edu | ${ }^{1}$ Department of Physics and Astronomy, Howard University, 2355 Sixth St NW, Washington, DC 20059, USA. 
In most studies of separation of $\mathrm{CO}_{2} / \mathrm{CH}_{4}$ at room temperature, the selectivities reported are smaller than 12 . One of the highest selectivities found has been achieved by the group of Palmer et al. [8]. They investigated different types of nanoporous carbons to separate $\mathrm{CO}_{2} / \mathrm{CH}_{4}$ mixtures at ambient temperature and pressure up to 10 MPa using Grand Canonical Monte Carlo (GCMC) simulations. They found that in the carbon slit pores, the selectivity reaches 12.1 for a mixture with $25 \% \mathrm{CH}_{4}$ and at a pressure of $3 \mathrm{MPa}$. In a mix with $50 \% \mathrm{CH}_{4}$ and pressure $4 \mathrm{MPa}$, the selectivity is 11 , whereas in a mixture with $75 \% \mathrm{CH}_{4}$ and pressure $6 \mathrm{MPa}$, the selectivity is 9.2.

Heuchel et al. [4] obtained $\Sigma\left(\mathrm{CO}_{2} / \mathrm{CH}_{4}\right)$ experimentally and theoretically on activated carbon A35/4 at 293 $\mathrm{K}$. They found selectivities in the range from 2.8 to 8.9 for various concentrations and pressures. Chen et al. [10], studied the $\mathrm{CO}_{2} / \mathrm{CH}_{4}$ selectivity in a MOF-505@ $\mathrm{GO}$ composite finding a value of 8.6 at $298 \mathrm{~K}$ and 100 $\mathrm{kPa}$. In a recent article, Szczȩśniak et al. [11] reported $\mathrm{CO}_{2} / \mathrm{CH}_{4}$ selectivity of 6.3 nand 5.8 in the $\mathrm{Cu}$-BTC MOF and Cu-BTC/GO10 respectively, at $298 \mathrm{~K}$ and 1 bar. Wang et al. [12] investigated $\mathrm{CO}_{2} / \mathrm{CH}_{4}$ separation in a pentagraphene (PG) nanosheet. They found that the selectivity is high when an electric field of 0.040 a.u. is applied. The effect of the electric field is to change the adsorbed $\mathrm{CO}_{2}$ from physisorption to chemisorption while not affecting the methane adsorption.

Other groups found more modest values for selectivity. Bastin et al. [1] examined a microporous MOF (MOF508b) for the separation and removal of $\mathrm{CO}_{2}$ from binary $\mathrm{CO}_{2} / \mathrm{CH}_{4}, \mathrm{CO}_{2} / \mathrm{N}_{2}$, and ternary $\mathrm{CO}_{2} / \mathrm{CH}_{4} / \mathrm{N}_{2}$ mixtures by fixed-bed adsorption at temperatures $303 \mathrm{~K}, 323 \mathrm{~K}$, and $343 \mathrm{~K}$. At $303 \mathrm{~K}$ for binary or ternary mixtures, the adsorption isotherms indicate that MOF-508b is moderately efficient for the removal of $\mathrm{CO}_{2}$. They reported that $\Sigma\left(\mathrm{CO}_{2} / \mathrm{CH}_{4}\right)$ and $\Sigma\left(\mathrm{CO}_{2} / \mathrm{N}_{2}\right)$ are in the range from 3 to 6 while decreasing with increasing temperature.

Liu et al. [5] investigated the adsorption and the selectivity of $\mathrm{CO}_{2}$ from $\mathrm{CO}_{2} / \mathrm{CH}_{4}, \mathrm{CO}_{2} / \mathrm{N}_{2}$ gas mixtures in oxygen-containing functionalized graphitic slit pores at a temperature of $298 \mathrm{~K}$ and pressures up to 300 bar using GCMC simulations. In their results, $\Sigma\left(\mathrm{CO}_{2} / \mathrm{CH}_{4}\right)$ was in the range from 2 to 5 .

Babarao et al. [2], predicted the value of $\Sigma\left(\mathrm{CO}_{2} / \mathrm{CH}_{4}\right)$ at room temperature in three different adsorbents: silicalite, $\mathrm{C}_{168}$ schwarzite, and IRMOF-1. By using GCMC, they found that the selectivity of $\mathrm{CO}_{2} / \mathrm{CH}_{4}$ is in the range from 2.0 to $3.2,2.0$ to 2.6 , and 3.0 to 5.0 for IRMOF-1, silicalite, and the $\mathrm{C}_{168}$ schwarzite, respectively. Peng et al. [9] investigated $\Sigma$ $\left(\mathrm{CO}_{2} / \mathrm{CH}_{4}\right)$ on ordered mesoporous carbon CMK-1 using GCMC at $\mathrm{T}=308 \mathrm{~K}$ and for different pressures and concentrations. Their results show that the highest selectivity of $\mathrm{CO}_{2}$ is 3.55 when $\mathrm{P}=7.0 \mathrm{MPa}$ and $\mathrm{y}_{\mathrm{CO}_{2}}=0.2$.
Other groups evaluated the selectivity at low temperatures, finding extremely high values. For instance, Gatica et al. [3], reported a numerical study of adsorption of $\mathrm{CO}_{2} / \mathrm{CH}_{4}$ on carbon nanohorns, finding selectivities growing from 6 to 25 for a temperature drop from $193 \mathrm{~K}$ to 143 $\mathrm{K}$. Maiga et al. [6, 7], estimated the selectivity of a $\mathrm{CO}_{2} / \mathrm{CH}_{4}$ binary mixture in MOFs and graphene using GCMC and the ideal adsorbed solution theory (IAST). For MOFs, they predicted that the selectivity increased from 2 to 250 for temperatures dropping from $300 \mathrm{~K}$ to $120 \mathrm{~K}$. For graphene at $40 \mathrm{~K}$, the selectivity estimated by GCMC-IAST reaches the extremely high value of $6.24 \times 10^{6}[6]$.

For practical reasons, it is vital to optimizing substrates capable of separation by adsorption at room temperature. In Fig. 1, we summarize the values of selectivities near 300 $\mathrm{K}$ reported by many groups.

We propose a simple substrate to reach high selectivity; it consists of graphene NRs placed over graphite. The NRs are assembled parallel to each other and the graphite surface. By tuning the separation between them, the edge style, and the distance between the NRs and graphite, we reach our objective.

Graphene NRs are quasi-one-dimensional carbon structures that can be obtained by cutting a graphene sheet into strips of a few nanometers width [13]. Graphene is itself a unique material with a high specific surface area of $\sim 2600 \mathrm{~m}^{2} / \mathrm{g}$, which is obtained from graphite through

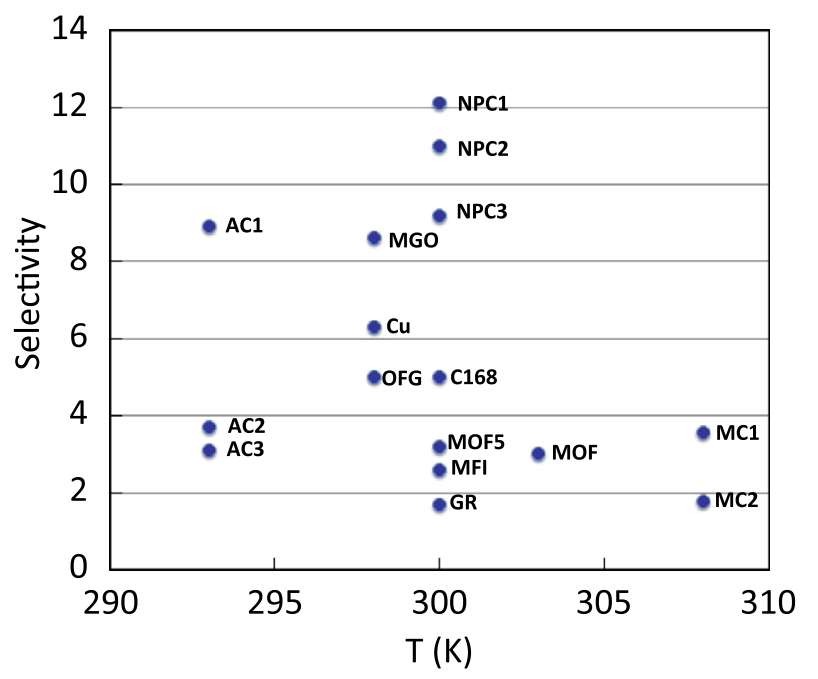

Fig. 1 Selectivity for $\mathrm{CO}_{2} / \mathrm{CH}_{4}$ near room temperature using different substrates: $\mathrm{C}_{168}$, MOF5 and MFI from Ref. [2]; MOF-508b (MOF) [1]; activated carbon at concentrations of $\mathrm{CO}_{2} \quad 90 \%$ (AC1), 50\% (AC2), and 20\% (AC3), respectively [4]; oxygen-containing functionalized graphitic slit pores (OFG) [5]; graphite (GR), from this work; nanoporous carbon at $25 \%$ concentration of $\mathrm{CH}_{4}$ and pressure $3 \mathrm{MPa}$ (NPC1), 50\%-4 MPa (NPC2), and 75\%-6MPa (NPC3) [8]; mesoporous carbon $\mathrm{CMK}-1$ at $20 \%$ concentration of $\mathrm{CO}_{2}$ and pressure 7.0 MPa (MC1), and 50\%-4.0 MPa (MC2) [9]; MOF-505@GO at $100 \mathrm{kPa}$ (MGO) [10]; Cu-BTC at 1 bar (Cu) [11] 
oxidation, exfoliation, and reduction [14]. Ever since the experimental isolation of graphene in 2004, significant research efforts have been focused on investigating the electronic and transport properties of its NRs. Several techniques have been developed to fabricate them. These include electron beam lithography and etching, chemical synthesis, and unzipping of carbon nanotubes [15]. The properties of graphene nano-ribbons can be tuned from metallic to semiconducting through changing the widths and the edge styles [16]. Paulla et al. [17] investigated graphene NRs for the sensing of carbon oxides. In their ab initio study, they found that pristine NRs may not be suitable for electrochemical sensing of carbon oxides with low concentration at room temperature; they suggest that adding an electric field can generate detectable coverages.

Although, in this work we do not address the technical aspects of keeping the graphene NRs fixed in place, we estimate that this can be accomplished by inserting spacers in between the NRs and the graphite surface, resembling the structure of MOFs or pillared graphene [18].

\section{Methods}

In this work, we compute, by MD simulations, the adsorption of a vapor mixture of $\mathrm{CO}_{2}$ and $\mathrm{CH}_{4}$ on the NRs/graphite substrate. We represent $\mathrm{CH}_{4}$ as a neutral spherical molecule with zero electric dipole and quadrupole moments. The $\mathrm{CH}_{4}-\mathrm{CH}_{4}$ intermolecular interaction energy is modeled as a Lennard Jones (LJ) potential. The $L J$ potential is given by,

$U_{U}=4 \epsilon\left[\left(\frac{\sigma}{r}\right)^{12}-\left(\frac{\sigma}{r}\right)^{6}\right]$

where $\epsilon$ and $\sigma$ are the energy and size LJ parameters, respectively, and $r$ is the distance between atoms. The $L J$ parameters for $\mathrm{CH}_{4}$ are $\epsilon=148.0 \mathrm{~K}$ and $\sigma=3.7 \AA$ adopted from Ref. [2]. We describe the $\mathrm{CO}_{2}$ molecule as a linear rigid body with three LJ sites and three partial charges placed on each atom. The carbon atom has a positive charge $q_{C}=0.576 e$, and the oxygen atoms have a negative charge $q_{O}=-0.288 e$. The bond length is $b=1.18 \AA \AA[2]$. The $\mathrm{CO}_{2}$ $-\mathrm{CO}_{2}$ intermolecular interaction energy is computed as a combination of the $L J$ and Coulomb potentials between partial charges. We adopt the LJ parameters from Ref. [2] (see Table 1).

For different species, the cross parameters are calculated by using the Lorentz-Bertholet combination rules [19],

$$
\begin{aligned}
\sigma_{i j} & =\frac{\sigma_{i i}+\sigma_{j j}}{2} \\
\epsilon_{i j} & =\sqrt{\epsilon_{i i} \epsilon_{j j}}
\end{aligned}
$$

Table 1 Lennard Jones parameters and partial charges

\begin{tabular}{llll}
\hline Adsorbate & $\epsilon(\mathrm{K})$ & $\sigma(\AA)$ & $q(e)$ \\
\hline $\mathrm{CH}_{4}$ & 148.0 & 3.7 & 0 \\
$\mathrm{C}_{\text {in } \mathrm{CO}_{2}}$ & 29.70 & 2.8 & +0.576 \\
$\mathrm{O}$ in $\mathrm{CO}_{2}$ & 83.00 & 3.0 & -0.288 \\
$\mathrm{C}$ in Graphene & 28.00 & 3.4 & 0 \\
\hline
\end{tabular}

In our simulations, we treat graphite as a continuous matter. The interaction energy between an adsorbate atom and the graphite substrate is evaluated by the Steele-10-4-3 potential [20] given by,

$$
\begin{aligned}
U_{\text {Steele }}(z)= & 2 \pi \epsilon \rho \sigma^{2} \Delta\left[\frac{2}{5}\left(\frac{\sigma}{z}\right)^{10}-\left(\frac{\sigma}{z}\right)^{4}\right. \\
& \left.-\frac{\sigma^{4}}{3 \Delta(z+0.61 \Delta)^{3}}\right]
\end{aligned}
$$

where $\epsilon$ and $\sigma$ are the $L J$ parameters and $z$ is the distance between the adatom and the graphite surface; the number of carbon atoms per unit volume in graphite is $\rho=0.114$ $\AA^{-3}$, and the separation distance between the layers of graphitic carbon is $\Delta=3.35 \AA$.

We describe the interaction between the NRs and the adsorbates by a sum of $L J$ potentials. The NRs are kept rigid and fixed during the simulations.

We run MD simulations at constant temperature by using the Nose-Hoover thermostat, which is based on the extended Lagrangian method. We run our simulations with the LAMMPS program, which stands for (Large-scale Atomic/Molecular Massively Parallel Simulator) [21, 22].

We set the simulation box with $160 \AA$ side lengths and periodic boundaries in $\mathrm{XY}$ directions. The box contains the right and left halves of two nanoribbons. The NRs are approximately $15-\mathrm{nm}$ wide and infinitely long due to the periodic boundary conditions (see Fig. 2). The top wall of the box is reflective, and the graphite surface is at the bottom of the cell. To create the NRs we place a graphene layer above the graphite substrate and delete the carbon atoms within a selected narrow region. By cutting the graphene layer in the $Y$ direction, we obtain zigzag NRs (ZRs), whereas cutting the graphene layer in the $X$ direction, gives armchair NRs (ARs). By manipulating the region where the carbon atoms are deleted, we get two different styles of edges, as shown in Fig. 3. The figure represents the ZRs in the upper row and ARs in the lower row with two different edge styles: VV and VB. In style VV, the vertexes are facing vertexes, and the bays are facing bays. For style VB where the vertexes are facing the bays, the gap opening is characterized by parameter a. For style $V \mathrm{~V}$, there are two parameters: $b$ and $c$, which are the distance from bay to bay and vertex to vertex, respectively. To fine-tune 


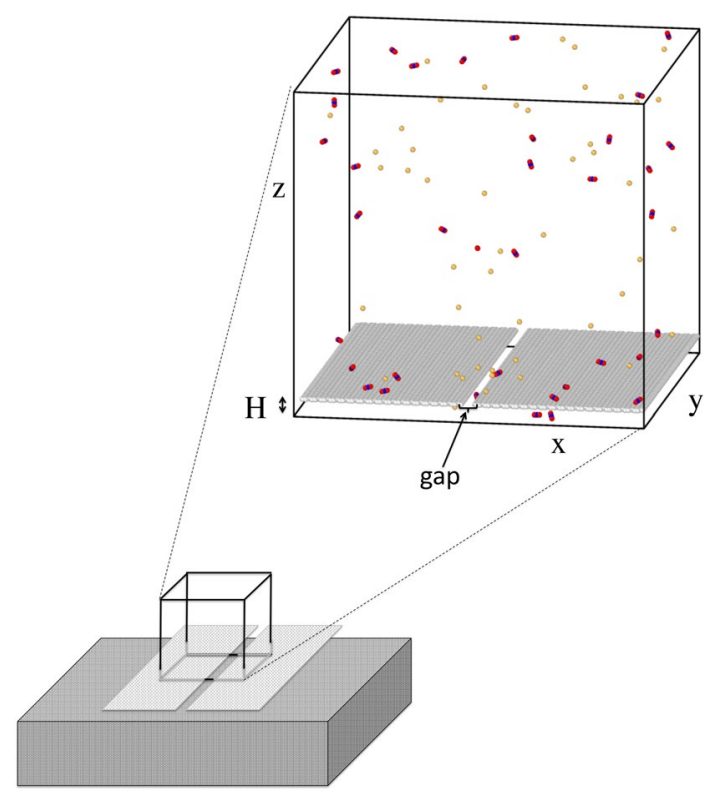

Fig. 2 Schematic picture of the nanoribbons over graphite and zoom of the simulation box

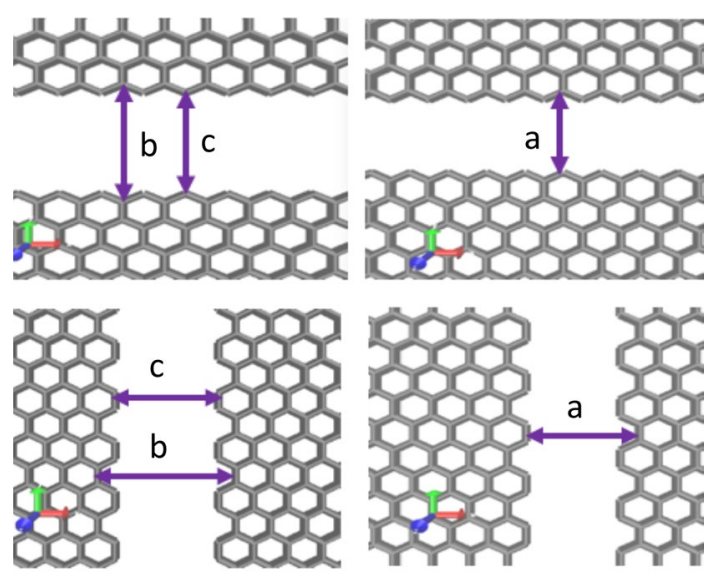

Fig. 3 Illustration of the edges of the graphene nanoribbons with styles zigzag-VV (top left), zigzag-VB (top right), armchair-VV (bottom left), armchair VB (bottom right)

the gap separation, we translate the NRs horizontally by tenths of angstroms.

The simulations are composed of two stages. First, we run a 1-million-steps $\mathrm{MD}$ of $50 \mathrm{CO}_{2}$ and $50 \mathrm{CH}_{4}$ molecules in the plain simulation box, to achieve a mixed vapor in equilibrium at $300 \mathrm{~K}$. In the second stage, we include the graphite substrate and the NRs in the simulation box and run MD for 5 million fs-timesteps. Molecules are attracted to graphene and pass through the slit forming layers on the graphite surface and the bottom of the ribbons.

The usual definition of selectivity given in Eq. 1 assumes that the system is in equilibrium with a vapor. That is not the case in MD simulations, where the process described is rather dynamic. Some molecules leave the vapor to occupy the region next to the substrate, while the total number of molecules in the box remains constant. Depending on the amount adsorbed, the molecules remaining in the vapor phase are only a few or even zero. For this reason, we redefine the selectivity as

$S=\frac{x_{\mathrm{CO}_{2}} / x_{\mathrm{CH}_{4}}}{y_{\mathrm{CO}_{2}} / y_{\mathrm{CH}_{4}}}$

where $x_{i}$ is the concentration of species $i$ in the adsorbed phase, and $y_{i}$ is the concentration in the simulation box. We compute $x_{i}$ by counting the number of molecules in a region of thickness $5-\AA \AA$ above the graphite surface. To calculate the selectivity, we use the average of $x_{\mathrm{CO}_{2}}$ and $x_{\mathrm{CH}_{4}}$ over the last million steps (last nanosecond).

To asses the kinetics of the process we define the relative rate of adsorption $R$ as,

$R=\frac{r_{\mathrm{CO}_{2}}}{r_{\mathrm{CH}_{4}}}$,

where $r_{\mathrm{CO}_{2}}$ and $r_{\mathrm{CH}_{4}}$ are the initial rate of adsorption for $\mathrm{CO}_{2}$ and $\mathrm{CH}_{4}$, respectively. The rates $r_{i}$ are calculated by a linear fit of the fraction adsorbed versus time on the first nanosecond.

\section{Results}

We studied various combinations of gap openings, NRsgraphite distances (from $6 \AA$ to $14 \AA$ ), and edge-styles (zigzag and armchair, VV and VB). In Table 2, we present the values of the selectivity and relative rate of adsorption for selected NRs with $S>2$ or $R>2$. In the cases where the adsorption of $\mathrm{CH}_{4}$ is zero during the first nanosecond interval, we report $R \rightarrow \infty$. Also, in the case of $x_{\mathrm{CH} 4}=0$ during the last nanosecond interval, the selectivity is infinite.

In Fig. 4 we show the results for the top-four selectivities. In the figure, we plot the adsorption of $\mathrm{CO}_{2}$ and $\mathrm{CH}_{4}$ on the graphite surface as a function of time. In the four cases, the graphene NRs are located at a distance $H=6 \AA$ above graphite. The maximum selectivity was obtained for VBedged ARs, with a vertex-bay distance of $7.4 \AA$ (see Fig. 4, top left). In this case, $\mathrm{CH}_{4}$ is wholly blocked out giving $S \rightarrow \infty$, and also due to zero $\mathrm{CH}_{4}$-adsorption in the first nanosecond, we obtain $R \rightarrow \infty$. The second top value of selectivity is 22 (Fig. 4 top right), for VV-edged ARs with parameters $\mathrm{b}=9.8 \AA$ and $\mathrm{c}=7.4 \AA$; the value of $R$ is 2.7. The third top value of selectivity is 21 , shown in Fig. 4 (bottom left). This case corresponds to VV-ARs with parameters $\mathrm{b}=14.8 \AA$ and $\mathrm{c}=12.3 \AA$. The fourth top value is $S=15$, 


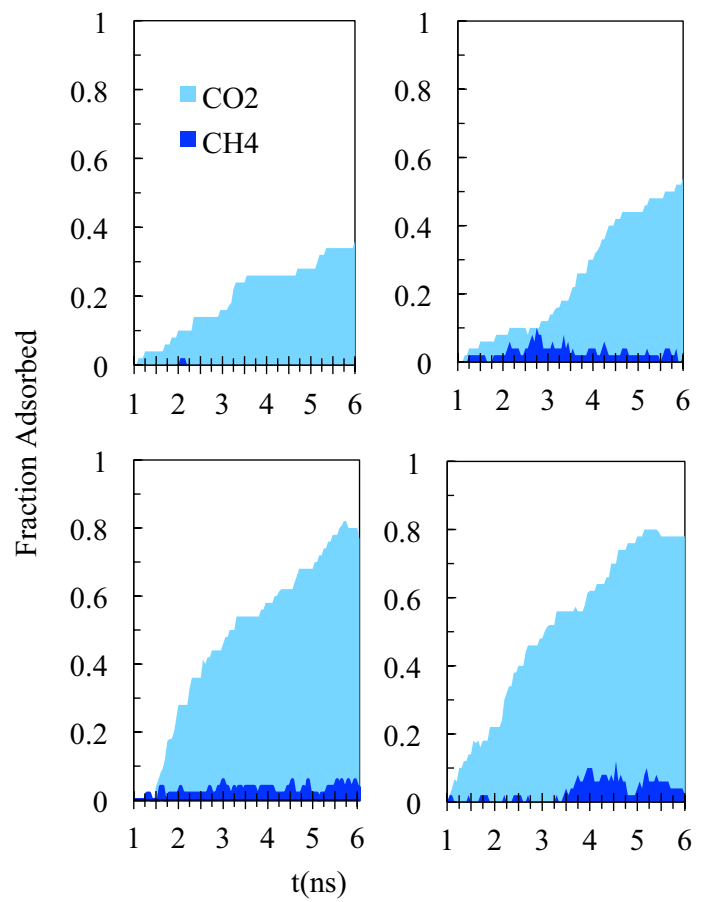

Fig. 4 Fraction adsorbed on graphite $x_{\mathrm{CO} 2}$ (lightblue) and $x_{\mathrm{CH} 4}$ (blue) on the graphite surface. The graphene ribbons are placed at a distance $\mathrm{H}=6 \AA$ above the graphite. The edge styles are armchair VB with parameter $\mathrm{a}=7.4 \AA$ (top left), armchair VV with $\mathrm{b}=9.8 \AA$ and $c=7.4 \AA$ (top right), armchair VV with $b=14.8 \AA$ and $c=12.3$ $\AA$ (bottom left) and zigzag VB with $\mathrm{a}=12.4 \AA$ (bottom right)

achieved with VB-ZRs with parameter $\mathrm{a}=12.4 \AA$. The last two cases also have high values of $R$ (14 and 18).

In all cases with $\mathrm{H}=6 \AA$ methane does not fit in the space between the NRs and graphite. However, for wider gaps (second, third, and fourth cases), methane molecules are adsorbed on graphite at the slit opening. To exemplify, we show snapshots of the simulation cell at $\mathrm{t}=6 \mathrm{~ns}$ in Figs. 5 and 6. Both cases correspond to $\mathrm{H}=6 \AA$ with gaps 7.4 $\AA$ (Fig. 5) and $14.8 \AA$ (Fig. 6). For the 14.8- $\AA$ gap, we see in Fig. 6 , a few $\mathrm{CH}_{4}$ molecules adsorbed on graphite right at the slit.

As a reference for comparison, we simulated adsorption of the mixture on plain graphite, obtaining $S=1.68$ and $R=1.98$. As it results, the selectivity and $R$ achieved with both ZRs and ARs are much higher than plain graphite.

When the distance between the graphene and graphite is higher than $8 \AA$ and the gap opening wider than $6.9 \AA$ in ZRs or $7.4 \AA$ on ARs, the values of $S$ and $R$ are not better than plain graphite. On the other hand, any smaller gap opening yields zero adsorption, because the slit then becomes too narrow for any molecule to pass. A distance $\mathrm{H}<6 \AA$ A results in zero adsorption as well, because the NRsgraphite separation becomes too thin to allow any of the molecules within.
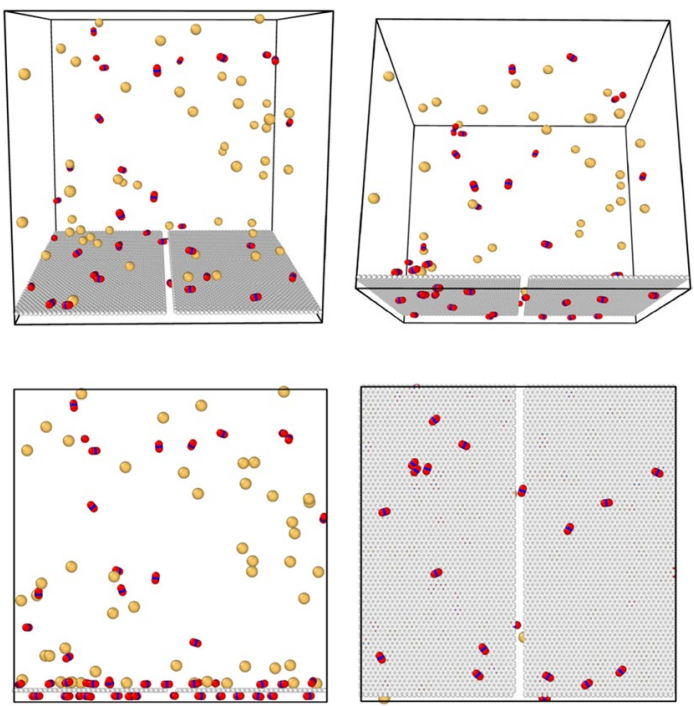

Fig. 5 Snapshots of the simulation at $\mathrm{t}=6 \mathrm{~ns}$, showing $\mathrm{CH}_{4}$ in yellow and $\mathrm{CO}_{2}$ in blue-red. The molecules are not in scale. The graphite surface is not shown for clarity. The panels display two perspective views (top row), a side view (bottom left) and a view from below the NRs (bottom right). The NRs' edges are armchair-VB with a 7.4- $\AA$ gap, and $\mathrm{H}=6 \AA$

The structure of the molecules play a crucial role; only the linear $\mathrm{CO}_{2}$ fits in the 6-Å narrow space between the NRs and graphite while the spherical $\mathrm{CH}_{4}$ does not. Moreover, the Van der Waals interaction between $\mathrm{CO}_{2}$ and graphite is stronger than $\mathrm{CH}_{4}$-graphite. In our simulations, we
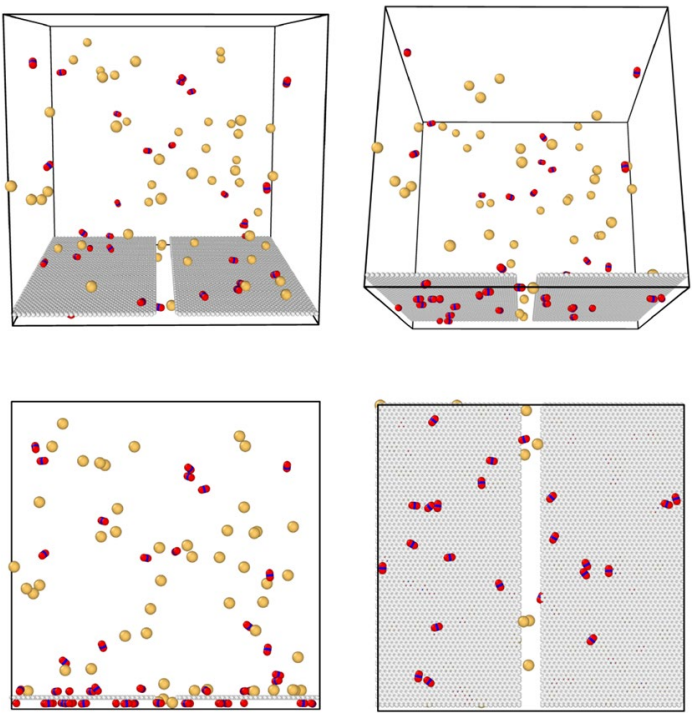

Fig. 6 Snapshots of the simulation at $\mathrm{t}=6 \mathrm{~ns}$, showing $\mathrm{CH}_{4}$ in yellow and $\mathrm{CO}_{2}$ in blue-red. The molecules are not in scale. The graphite surface is not shown for clarity. The panels display two perspective views (top row), a side view (bottom left) and a view from below the NRs (bottom right). The NRs' edges are armchair-VV with a $14.8-\AA$ gap, and $\mathrm{H}=6 \AA$ 
Table 2 Selectivity and relative rate of adsorption for selected slit openings

\begin{tabular}{|c|c|c|c|c|}
\hline$\overline{H(\AA)}$ & Style & $\operatorname{Gap}(\AA ̊)$ & $S$ & $R$ \\
\hline 6 & $A \vee B$ & 7.4 & $\infty$ & $\infty$ \\
\hline 6 & $A V V$ & 9.8 & 22 & 2.7 \\
\hline 6 & $A V V$ & 14.8 & 21 & 14 \\
\hline 6 & Z VB & 12.4 & 15 & 18 \\
\hline 6 & ZVV & 14.2 & 11 & 9.6 \\
\hline 6 & Z VB & 6.9 & 8.8 & 12 \\
\hline 12 & $A \vee B$ & 7.4 & 8.6 & 4.0 \\
\hline 12 & Z VB & 6.9 & 8.4 & 14 \\
\hline 6 & A VV & 12.3 & 7.9 & 11 \\
\hline 10 & $A \vee B$ & 7.4 & 7.5 & 8.5 \\
\hline 14 & $A \vee B$ & 7.4 & 7.0 & 4.3 \\
\hline 6 & ZVV & 9.8 & 6.5 & 6.9 \\
\hline 10 & Z VB & 6.9 & 6.5 & 2.7 \\
\hline 14 & Z VB & 6.9 & 6.1 & $\infty$ \\
\hline 8 & Z VB & 6.9 & 5.9 & 2.7 \\
\hline 8 & $A \vee B$ & 7.4 & 5.5 & 3.8 \\
\hline 14 & Z VB & 12.4 & 1.9 & 3.1 \\
\hline 14 & ZVV & 9.8 & 1.8 & 3.3 \\
\hline
\end{tabular}

computed the average adsorption energy between graphite and $\mathrm{CO}_{2}, \mathrm{E}_{\mathrm{G}-\mathrm{CO}_{2}}=3.76 \mathrm{Kcal} / \mathrm{mol}$, while $\mathrm{E}_{\mathrm{G}-\mathrm{CH}_{4}}=2.64$ $\mathrm{Kcal} / \mathrm{mol}$. As a result, the selectivity for $\mathrm{CO}_{2}$ is high due to a combination of energetic and steric effects.

The kinetics of adsorption is also greatly improved. In plain graphite, the initial uptake of $\mathrm{CO}_{2}$ is just twice as fast as for $\mathrm{CH}_{4}$. In the NRs/graphite substrate, the relative rate $R$ reaches 18 and 14 for ZRs and ARs, respectively.

\section{Discussion}

Our findings show that the separation of a carbon dioxide and methane mixture on NRs-Graphite at room temperature is significantly enhanced. All the NRs edges tested in this work are adequate for filtering carbon dioxide.

The mechanisms involved in the performance of the filter combine energetics, kinetics, and steric aspects of adsorption. The energetics works because $\mathrm{CO}_{2}$ is more intensively attracted to the substrate; the kinetic factor arises from the higher initial rate of adsorption of $\mathrm{CO}_{2}$ even in non-blocking cases. There is an interplay of adsorption and diffusion above and below the graphene NRs and on graphite. $\mathrm{CO}_{2}$ adsorbs first on top of the graphene, followed by $\mathrm{CH}_{4}$. Then, $\mathrm{CO}_{2}$ diffuses through the gap and forms monolayer films on the graphite surface and below the NRs. If the distance between the NRs and graphite is $6 \AA, \mathrm{CH}_{4}$ is completely blocked out even with wide enough NRs gap openings. This is a consequence of the overlap of the repulsive cores of the $\mathrm{CH}_{4}$ -graphene and $\mathrm{CH}_{4}$-graphite interactions that results in a total repulsion of methane. In the non-blocking cases, $\mathrm{CH}_{4}$ molecules would start adsorbing on graphite later than $\mathrm{CO}_{2}$.

The optimal parameters for the filter are a NRs-graphite separation of $6 \AA$, armchair-VB edge style, and gap opening of $7.4 \AA$. However, slightly larger separations and openings and other edge styles are also effective.

The results of this work involve several approximations, as it is the case in all computer simulations. The most crucial estimate is the calculation of the interaction energy and forces. The exact count of this dispersion energy is an impossible task that requires the solution of Schrödinger equation for the system. In our model, the interaction between the $\mathrm{CO}_{2}$ molecules and the graphene NRs is computed using the Lorentz-Bertholet combination rules for the $L J$ parameters. The minimum energy of $\mathrm{CCO}_{2}$ molecule on a graphene sheet obtained with our model is $-3.699 \mathrm{kcal} /$ mole. This energy corresponds to a molecule parallel to the graphene plane, with the carbon atom on top of the line joining two graphene's carbon atoms and the oxygen atoms on top of the center of the hexagons. The distance between the molecule and the graphene plane is $3.14 \AA$. These values coincide with the results of DFT calculations of $\mathrm{CO}_{2}$ on polycyclic aromatic hydrocarbons [23]. Other approximations include the rigidity of the $\mathrm{NRs}$ and $\mathrm{CO}_{2}$ molecules, the continuous model of the graphite substrate, and the spherical model of the $\mathrm{CH}_{4}$ molecule. The effect of these components of the model will be considered in further work.

As mentioned above, although we have not addressed the technical aspects of keeping the graphene NRs fixed in place, we would estimate that this can be accomplished by inserting organic molecules as pillars resembling the structure of pillared graphene [18]. However, the optimal separation of $6 \AA$ may be too small to be achieved by a pillared structure. In this case, we would envision a different approach similar to the graphite intercalation compounds [24]. Of course, the technicality is far from the scope of our work. However we must say that adding such pillars or spacers would affect the adsorption rates but not the fact that methane is blocked out when the separation is $6 \AA$ wide. Finally, the reader may argue that the proposed substrate lacks the capacity for storage. That is true, and we would like to emphasize that our results pertain to the filtering rather than the storage. As such, the filter should be combined with a supplementary substrate for that purpose.

Acknowledgements This work used the Extreme Science and Engineering Discovery Environment (XSEDE) through allocation TG-DMR180036. 


\section{Compliance with ethical standards}

Conflict of interest The authors declare that they have no conflict of interest.

\section{References}

1. Bastin I, Barcia PS, Hurtado EJ, Silva JAC, Rodrigues AE, Chen B (2008) A microporous metal organic framework for separation of $\mathrm{CO}_{2} / \mathrm{N}_{2}$ and $\mathrm{CO}_{2} / \mathrm{CH}_{4}$ by fixed-bed adsorption. J Phys Chem C 112:1575

2. Babarao R, Hu Z, Jiang J (2007) Storage and separation of $\mathrm{CO}_{2}$ and $\mathrm{CH}_{4}$ in silicalite, $\mathrm{C}_{168}$ schwarzite, and IRMOF-1: a comparative study from Monte Carlo simulation. Langmuir 23:659

3. Gatica SM, Nekhai A, Scrivener A (2016) Adsorption and gas separation of molecules by carbon nanohorns. Molecules 21:662

4. Heuchel M, Davies GM, Buss E, Seaton NA (1999) Adsorption of carbon dioxide and methane and their mixtures on an activated carbon: simulation and experiment. Langmuir 15:8695

5. Liu Y, Wilcox J (2013) Molecular simulation studies of $\mathrm{CO}_{2}$ adsorption by carbon model compounds for carbon capture and sequestration applications. Environ Sci Technol 47:95

6. Maiga SM (2016) Ph.D. Dissertation, Howard University Washington, D.C

7. Maiga SM, Medina MA, Durodola OJ, Gatica SM (2013) Simulations of adsorption of $\mathrm{CO}_{2}$ and $\mathrm{CH}_{4}$ in MOFs: effect of the size andcharge distribution on the selectivity. J Low Temp Phys 172:274

8. Palmer JC, Moore JD, Roussel TJ, Brennan JK, Gubbins KE (2011) Adsorptive behavior of $\mathrm{CO}_{2}, \mathrm{CH}_{4}$ and their mixtures in carbon nanospace: a molecular simulation study. Phys Chem Chem Phys 13:3985

9. Peng X, Cao D, Zhao J (2009) Grand canonical Monte Carlo simulation of methane-carbon dioxide mixtures on ordered mesoporous carbon CMK-1. Sep Purif Technol 68:50

10. Chen Y, Lv D, Wu J, Xiao J, Xi H, Xia Q, Li Z (2017) A new MOF$505 @ G O$ composite with high selectivity for $\mathrm{CO}_{2} / \mathrm{CH}_{4}$ and $\mathrm{CO}_{2}$ $\mathrm{N}_{2}$ separation. Chem Eng J 308:1065. https://doi.org/10.1016/j. cej.2016.09.138

11. Szczęśniak B, Choma J (2020) Graphene-containing microporous composites for selective $\mathrm{CO}_{2}$ adsorption. Microporous and Mesoporous Mater 292:109761. https://doi.org/10.1016/j.micro meso.2019.109761

12. Wang $M$, Zhang Z, Gong Y, Zhou S, Wang J, Wang Z, Wei S, Guo W, LuX (2020) Penta-graphene as a promising controllable $\mathrm{CO}_{2}$ capture and separation material in an electric field. Appl Surf Sci 502:144067. https://doi.org/10.1016/j.apsusc.2019.144067

13. Soroko VA, Batrakov KG, Chrnozatonskii LA (2014) Edge-modified zigzag-shaped graphene nanoribbons: structure and electronic properties. Phys Solid State 56:2135

14. Gadipelli S, Xiao Guo Z (2014) Graphene-based materials: synthesis and gas sorption, storage and separation. Prog Mater Sci 69:1

15. Orlof A, Ruseckas J, Zozoulenko IV (2013) Effect of zigzag and armchair edges on the electronic transport in single-layer and bilayer graphene nanoribbons with defects. Phys Rev B 88:125409

16. Yin W, Xie Y, Liu L, Chen Y, Wang R, Wei X, Zhong J, Lau L (2013) Atomic structure and electronic properties of folded graphene nanoribbons: a first-principles study. J Appl Phys 113:173506

17. Paulla KK, Farajian AA (2013) Concentration effects of carbon oxides on sensing by graphene nanoribbons: ab initio modeling. J Phys Chem C 117(24):12815. https://doi.org/10.1021/ jp312711s

18. Tylianakis GKDE, Froudakis GE (2008) Pillared graphene: a new 3-D network nanostructure for enhanced hydrogen storage. Nano Lett 8(10):3166. https://doi.org/10.1021/nl801417w PMID: 18800853

19. Bruch LW, Cole MW, Zaremba E (1997) Physical adsorption: forces and phenomena. Clarendon Press, Oxford

20. Steele WA (1973) The physical interaction of gases with crystalline solids: I. Gas-solid energies and properties of isolated adsorbed atoms. Surf Sci 36:317. https://doi.org/10.1016/00396028(73)90264-1

21. Plimpton S (1995) Fast parallel algorithms for short-range molecular dynamics. J Comput Phys 117:1

22. LAMMPS website. http://lammps.sandia.gov. Accessed 10 Aug 2019

23. Umadevi D, Sastry GN (2011) Molecular and ionic interaction with graphene nanoflakes: a computational investigation of $\mathrm{CO}_{2}, \mathrm{H}_{2} \mathrm{O}, \mathrm{Li}, \mathrm{Mg}, \mathrm{Li}+$, and $\mathrm{Mg} 2+$ interaction with polycyclic aromatic hydrocarbons. J Phys Chem C 115(19):9656. https:// doi.org/10.1021/jp201578p

24. Dresselhaus MS, Dresselhaus G (1981) Intercalation compounds of graphite. Adv Phys 30(2):139. https://doi.org/10.1080/00018 738100101367

Publisher's Note Springer Nature remains neutral with regard to jurisdictional claims in published maps and institutional affiliations. 